\title{
Influence of the heat source location on the stability of the solution to the Cauchy problem
}

\author{
Magda Joachimiak ${ }^{1 *}$, and Michat Ciałkowski ${ }^{1}$ \\ ${ }^{1}$ Institute of Thermal Engineering, Poznan University of Technology, 60-965 Poznan, Poland
}

\begin{abstract}
In this paper the solution to the Cauchy-type inverse problem for the Laplace's equation is presented. A modified Tikhonov regularization was applied here. The regularization parameter was chosen using the Morozov principle. The relation between the location of the heat source (function singularity) and the stability of the solution to the inverse problem was analyzed. Variable thermal loads in the area were simulated by changing the location of heat sources along two boundaries of the rectangle calculation domain.
\end{abstract}

\section{Introduction}

Inverse problems are numerically ill-conditioned [1]. They are widely applied in technical issues [2-14]. In the reference paper [15] authors examined the solution to the boundary inverse problem and the quasi-Cauchy problem for the Laplace's equation using the Chebyshev polynomials. The Cauchy-type inverse problem was investigated using the Chebyshev polynomials in the reference paper [16]. In this paper authors expand the scope of research work presented in reference papers [15-17] by including problems concerning the impact of the location of the heat source on the stability of the solution to the Cauchy problem.

\section{Calculation model}

Given is the Laplace's equation

$$
\frac{\partial^{2} T}{\partial x^{2}}+\frac{\partial^{2} T}{\partial y^{2}}=0
$$

in the domain $\Omega=\langle-1,1\rangle \times\langle-1,1\rangle$ with the boundary conditions (fig. 1)

$$
\begin{array}{lc}
\Gamma_{2}: & T(x, y=1)=T_{\Gamma_{2}}(x) \\
\Gamma_{3}: & T(x=-1, y)=T_{\Gamma_{3}}(y) \\
\Gamma_{3}: & \frac{\partial T(x=-1, y)}{\partial n}=q_{\Gamma_{3}}(y) \\
\Gamma_{4}: & T(x, y=1)=T_{\Gamma_{2}}(x)
\end{array}
$$

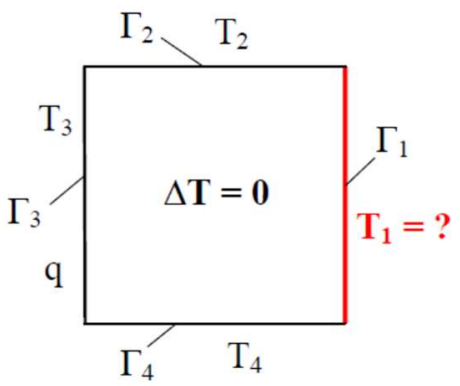

Fig. 1. Calculation domain with marked boundary conditions [16]

The sought temperature distribution on the boundary $\Gamma_{1}$ was approximated by the linear combination of the Chebyshev polynomials. To determine the distribution of temperature on the boundary $\Gamma_{1}$, the functional of the following form was minimized

$$
\begin{aligned}
J= & \int_{\Gamma_{2}}\left(T_{o b l, \Gamma_{2}}-T_{m, \Gamma_{2}}\right)^{2} d s+\int_{\Gamma_{3}}\left(T_{o b l, \Gamma_{3}}-T_{m, \Gamma_{3}}\right)^{2} d s+ \\
& +\int_{\Gamma_{3}}\left(\frac{\partial T_{o b l, \Gamma_{3}}}{\partial n}-q_{m, \Gamma_{3}}\right)^{2} d s+\int_{\Gamma_{4}}\left(T_{o b l, \Gamma_{4}}-T_{m, \Gamma_{4}}\right)^{2} d s
\end{aligned}
$$

The Cauchy-type inverse problem is numerically illposed. Slight disturbance to input data results in a significant change of the solution. Therefore, its regularization is needed. The modified Tikhonov regularization was applied.

$$
J_{\alpha}=J+\alpha^{2} I=J+\alpha^{2} \int_{\Gamma_{1}}\left((T)^{2}+\left(\frac{\partial T}{\partial y}\right)^{2}\right) d \Gamma_{1}
$$

Regularization parameter $\alpha$ was chosen with the use of the Morozov principle [18]. This calculation model is described in detail in the reference paper [16].

\footnotetext{
*Corresponding author: magda.joachimiak@put.poznan.pl
} 


\section{Numerical examples}

In this paper authors analyzed the impact of the location of the heat source (singularity of the function $\mathrm{f}$ at the point $x=a$ and $y=b$ ) on the distribution of temperature on the boundary $\Gamma_{1}$ for the function

$$
f=\ln \left((x-a)^{2}+(y-b)^{2}\right)
$$

Variability of the sought temperature (on the boundary $\Gamma_{1}$ ) depends on the location of the function singularity for the given for numerical tests function of temperature. The location of heat source is outside of domain $\Omega$. Heat flux density was next randomly disturbed by values reaching up to $0.01 q$. Calculations were performed for singularities located along the boundary $\Gamma_{3}(a=-1.2, b$ from -1 to 1.2 , fig. 2$)$ and along the boundary $\Gamma_{2}(b=$ $1.2, a$ from -1 to 1 , fig. 3 ). The norm $N_{\max }$ was calculated as per the formula

$$
N_{\max }=\frac{\max \left|T_{\Gamma 1_{-} C}-T_{\Gamma 1_{-} A}\right|}{\max \left|T_{\Gamma 1_{-} A}\right|}
$$

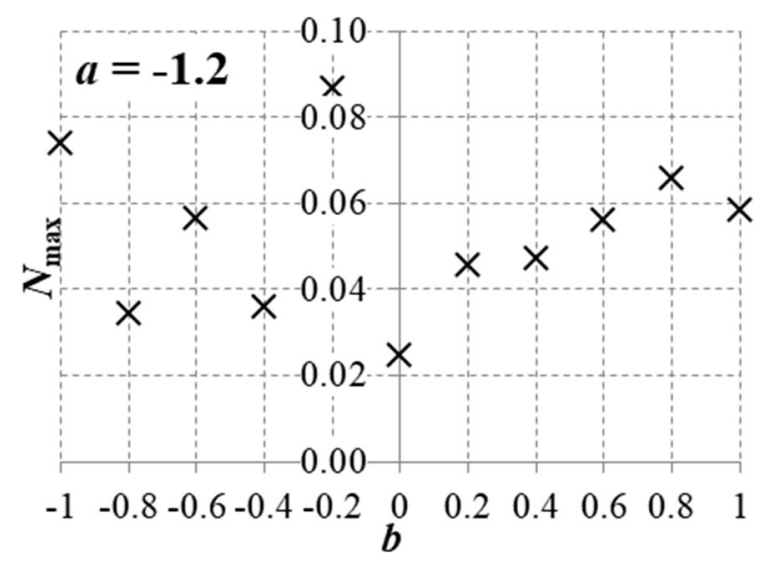

Fig. 2. Values of the norm $N_{\max }$ for $a=-1.2$ and $b$ from -1 to 1.2

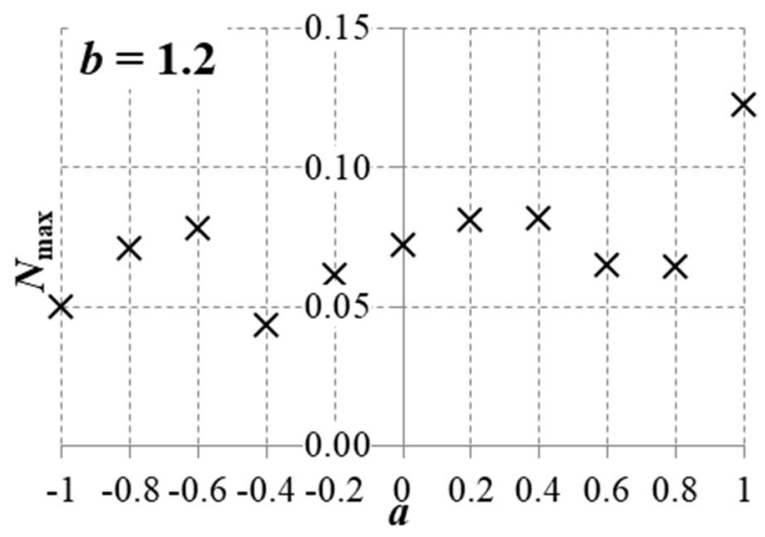

Fig. 3. Values of the norm $N_{\max }$ for $b=1.2$ and $a$ from -1 to 1

The highest value of the norm $N_{\max }$ was obtained for $a=$ $1, b=1.2$. It is the point located as close as possible to the boundary $\Gamma_{1}$. It results from calculations that shifting the singularity towards the boundary $\Gamma_{1}$ results in obtaining the solution being more sensitive to disturbances to the set values.

\section{Conclusion}

This paper presents the solution to the inverse problem for the Laplace's equation. To obtain a stable solution authors applied the modified Tikhonov regularization. The impact of shifting of the heat source along the boundaries $\Gamma_{3}$ and $\Gamma_{2}$ on the solution was investigated. The highest value of the norm $N_{\max }=1.22 \cdot 10^{-1}$ was obtained for $a=1$ and $b=1.2$, while the lowest norm $N_{\max }=2.48 \cdot 10^{-2}$ was achieved for $a=-1.2$ and $b=0$. Conducted numerical simulations in the function of the heat source location are characterized by a stable solution to the inverse problem and a low sensitivity to disturbed input data.

\section{Nomenclature}

$$
\begin{aligned}
& J_{\alpha} \quad-\text { regularization functional } \\
& \text { max - maximal value } \\
& N_{\text {max }}-\text { norm } \\
& T \text { - temperature, } \mathrm{K} \\
& \alpha \quad \text { - regularization parameter } \\
& \Gamma \quad \text { - boundary of the domain } \Omega \text {, } \\
& \left(\Gamma=\Gamma_{1} \cup \Gamma_{2} \cup \Gamma_{3} \cup \Gamma_{4}\right) \\
& \Omega \text { - calculation domain } \\
& \text { Subscript: } \\
& \text { C - calculated value } \\
& A \text { - analytical solution }
\end{aligned}
$$

\section{References}

1. J. Hadamard, Sur les problèmes aux dérivéespartielles et leur signification physique (Princeton Univ. Bull. 1902)

2. Ł. Brodzik, and A. Frąckowiak, Int. J. Numer. Methods Heat Fluid Flow 30, 2989, (2020) 